\title{
BRIEF COMMUNICATION OPEN Psychomotor function measured via online activity predicts motor vehicle fatality risk
}

\author{
Tim Althoff ${ }^{1}$, Eric Horvitz ${ }^{2}$ and Ryen W. White ${ }^{2}$
}

Impaired psychomotor performance severely increases the risk of fatal and non-fatal car accidents. However, we currently lack methods to continuously and non-intrusively monitor psychomotor performance. We show we can estimate psychomotor function at population scale from 16 billion observations of typing speeds during the input of web search queries. We show that these estimates exhibit diurnal variation with a substantial increase during typical sleep times, matching published accident risk rates. Further, we show that psychomotor impairment, as measured by keystroke timing, predicts motor vehicle fatality risk on a population level (Spearman $\rho=0.61 ; p \ll 10^{-10}$ ). The methods and results highlight a promising direction of harnessing ambient streams of data, such as patterns of interactions with devices, as large-scale sensors to continuously and non-intrusively monitor human psychomotor performance at population scale.

npj Digital Medicine (2018)1:20173; doi:10.1038/s41746-017-0003-3

\section{INTRODUCTION}

Motor vehicle crashes are responsible for 1.25 million deaths annually and are the leading cause of death for people of ages 15-29. ${ }^{1}$ The risk of car crashes based on operator error increases significantly with insufficient sleep. ${ }^{2,3}$ Recent advances in inferring psychomotor function using measures of typing speed of queries during web search enable population-scale estimation of psychomotor impairment. ${ }^{4}$ We examine whether psychomotor performance as indicated by slower typing speeds during web search predicts population-level motor vehicle fatalities by locale.

\section{RESULTS}

County-level average keystroke timing is strongly correlated with motor vehicle fatalities across 2723 counties (Spearman $\rho=0.61$; $p \ll 10^{-10}$; Fig. 1a). Controlling for potential confounding factors in a multivariate linear regression, keystroke timing remains a statistically significant predictor of motor vehicle fatalities ( $p$ « $10^{-10}$; $t$-test; Adj. $\left.R^{2}=0.554 ; N=2,555\right)$. This correlation is further illustrated through the ordering by keystroke timing for the five largest California counties by population (Fig. 1b). For example, San Bernadino County has the highest average keystroke times and also the largest number of deaths due to traffic accidents (11.57 per population of 100,000). Additionally, the diurnal variation in keystroke timing matches that of published accident risk rates with a substantial increase during typical sleep times. ${ }^{5}$

\section{DISCUSSION}

Interactions with a web search engine enable inferences about accident risk on a national scale. The study was limited by its cross-sectional and correlational design. Search queries and accident risk data were collected during different time periods and included subjects do not necessarily overlap. However, fatality rates have been highly correlated from year to year
(Supplementary Figs. 1 and 2), suggesting that our statistical analyses are insensitive to the shift in time of the observational window.

Beyond uses in population-level studies, patterns of interaction with online services may enable continuous, longitudinal monitoring of individuals for fatigue and other forms of psychomotor impairment. Future research directions include investigation of the predictive power of keystroke timing on accident risk on an individual level.

\section{METHODS}

Centers for Disease Control and Prevention mortality statistics ${ }^{6}$ from 2007 to 2013 (the most recent year available) were used to compute populationnormalized, annual mean motor vehicle mortality rates on a county level. Year-to-year fatality rates have been stable over time within individual counties (Supplementary Figs. 1 and 2).

Average keystroke timings across all US counties over a 4-month interval (April-July 2016) were computed from archival, de-identified search query logs from the Bing web search engine of data routinely collected for improving search results and permitted through Bing's Terms of Service. Only queries from desktop and notebook computers were used and queries from mobile devices were excluded. Keystroke timing is defined as the time in milliseconds between two key-down events and is estimated from consecutive search engine requests as detailed in Althoff et al. ${ }^{4}$ Search engine requests from counties with less than 10,000 keystrokes in total (4.7\%) were excluded. The sample includes 16.1 billion timed keystrokes over 2723 counties. Keystroke timing estimates have high precision due to the large number of keystrokes from each individual country (e.g., 393 million keystrokes from Los Angeles county alone).

To control for potential confounding, a multivariate linear regression analysis was performed that included the following factors ${ }^{6}$ (using all 2555 counties with complete records out of 2723 total counties): age (fraction of population below 18 or above 65 ), gender (\% female), education (\% graduated from high school, \% with some college education), income (median household income), unemployment (\% civilian labor force that is unemployed and seeking work), long commutes (\% commuters, among

\footnotetext{
${ }^{1}$ Stanford University, Stanford, CA, USA and ${ }^{2}$ Microsoft, One Microsoft Way, Redmond, WA 98052, USA

Correspondence: Tim Althoff (althoff@cs.stanford.edu)
}

Received: 12 July 2017 Revised: 28 August 2017 Accepted: 30 August 2017

Published online: 15 January 2018 
(A) 35

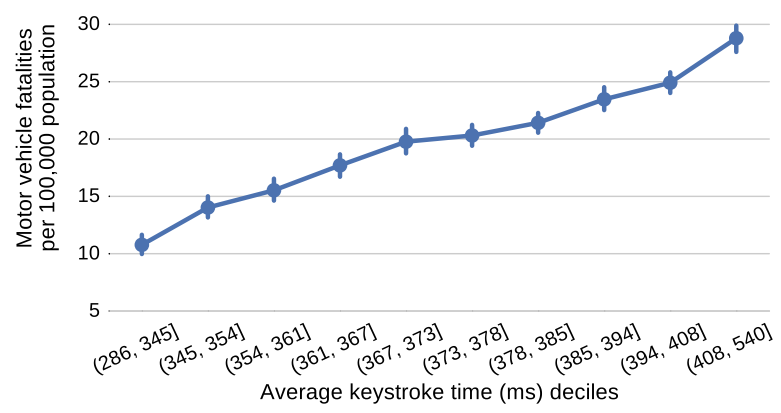

(B)
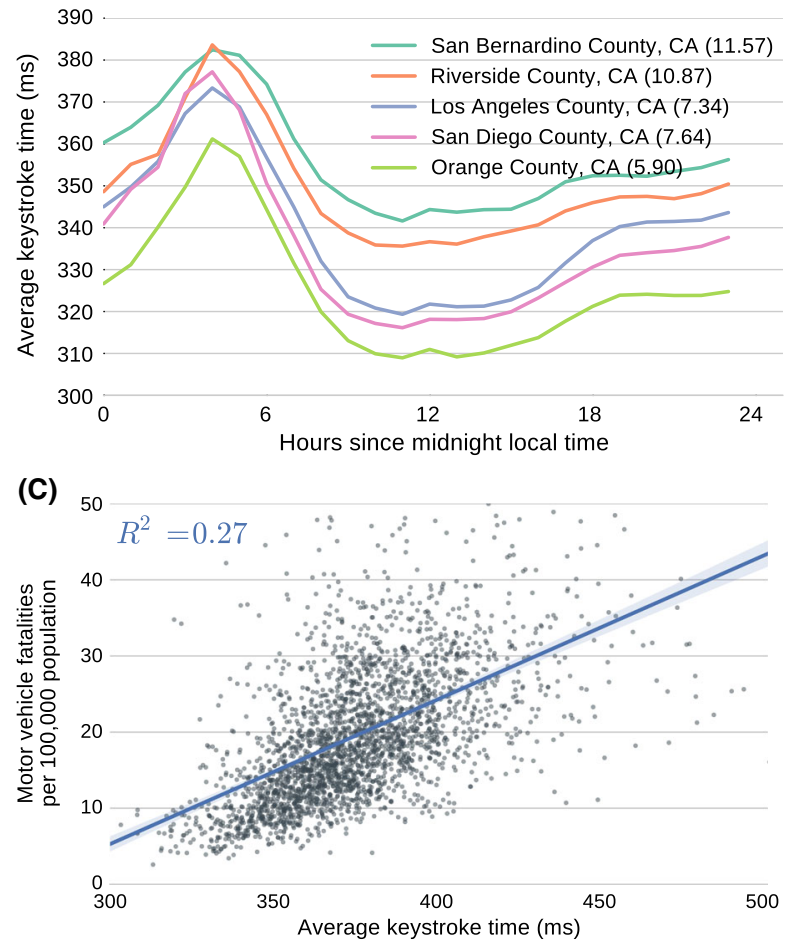

Fig. 1 a Keystroke timing is strongly correlated with the populationnormalized number of motor vehicle fatalities across 2723 US counties. b Average keystroke timing over time for five example counties in California. The number in parentheses after each county name refers to the population-normalized number of motor vehicle fatalities. c Number of motor vehicle fatalities (population-normalized) by average keystroke timing across 2723 US counties

those who commute to work alone, who drive longer than 30 min to work each day), fraction of population living in rural area, insufficient sleep (\% reporting sleeping less than $7 \mathrm{~h}$ per night), fraction of population reporting excessive drinking, and fraction of traffic accident deaths with alcohol involvement.

This study was conducted in accordance with guidance from the Microsoft Ethics Review Board.

Data availability

The data that support the findings of this study are available from Microsoft but restrictions apply to the availability of the data. Data are available from the authors on reasonable request and with permission of Microsoft.

\section{ACKNOWLEDGEMENTS}

This research was conducted during an internship at Microsoft Research. The authors thank Jure Leskovec and Jamie Zeitzer for helpful comments and discussions. This work was supported by Microsoft. The sponsor had no role in the design and conduct of the study; collection, management, analysis, and interpretation of the data; preparation, review, or approval of the manuscript; and decision to submit the manuscript for publication.

\section{AUTHOR CONTRIBUTIONS}

T.A. had full access to all of the data in the study and takes responsibility for the integrity of the data and the accuracy of the data analysis. All authors are equally responsible for the Concept and design, Acquisition, analysis, or interpretation of data:, Drafting of the manuscript, and also critical revision of the manuscript for important intellectual content. Statistical analysis: T.A. Obtained funding: R.W.W. and E.H. Administrative, technical, or material support: R.W.W. and E.H.

\section{ADDITIONAL INFORMATION}

Supplementary information accompanies the paper on the npj Digital Medicine website (https://doi.org/10.1038/s41746-017-0003-3).

Competing interests: The authors declare no competing financial interests.

Publisher's note: Springer Nature remains neutral with regard to jurisdictional claims in published maps and institutional affiliations.

Change history: The original version of this Article had an incorrect Article number of 3 and an incorrect Publication year of 2017. These errors have now been corrected in the PDF and HTML versions of the Article.

\section{REFERENCES}

1. World Health Organization. Global Status Report On Road Safety 2015 (WHO, Geneva, 2015).

2. Dinges, D. F. An overview of sleepiness and accidents. J. Sleep Res 4, 4-14 (1995).

3. Martiniuk, A. L. et al. Sleep-deprived young drivers and the risk for crash: the drive prospective cohort study. JAMA Pediatr. 167, 647-655 (2013).

4. Althoff, T., Horvitz, E., White, R. W. \& Zeitzer, J. harnessing the web for populationscale physiological sensing: a case study of sleep and performance. In Proc. ACM International World Wide Web Conference (WWW) (International World Wide Web Conference Committee, Perth, 2017).

5. Folkard, S. Black times: temporal determinants of transport safety. Accid. Anal. Prev. 29, 417-430 (1997).

6. Robert Wood Johnson Foundation and the University of Wisconsin Population Health Institute. County health rankings: motor vehicle crash deaths. http://www. countyhealthrankings.org/measure/motor-vehicle-crash-deaths. Accessed 1 Oct 2016.

(c) This article is licensed under a Creative Commons Attribution 4.0 By International License, which permits use, sharing, adaptation, distribution and reproduction in any medium or format, as long as you give appropriate credit to the original author(s) and the source, provide a link to the Creative Commons license, and indicate if changes were made. The images or other third party material in this article are included in the article's Creative Commons license, unless indicated otherwise in a credit line to the material. If material is not included in the article's Creative Commons license and your intended use is not permitted by statutory regulation or exceeds the permitted use, you will need to obtain permission directly from the copyright holder. To view a copy of this license, visit http://creativecommons. org/licenses/by/4.0/.

(C) The Author(s) 2018 